\title{
Effects of fructo-oligosaccharide-supplemented infant cereal: a double-blind, randomized trial
}

\author{
Nancy Moore ${ }^{1 *}$, Cewin Chao ${ }^{1}$, Li-Ping Yang ${ }^{1}$, Heidi Storm ${ }^{2}$, Maria Oliva-Hemker ${ }^{1}$ and \\ Jose M. Saavedra ${ }^{1,2}$ \\ ${ }^{1}$ Department of Pediatrics, Johns Hopkins University School of Medicine, Baltimore, MD, USA \\ ${ }^{2}$ Nestlé Nutrition Institute, Glendale, CA, USA
}

(Received 6 January 2003 - Revised 24 April 2003 - Accepted 29 May 2003)

\begin{abstract}
Fructo-oligosaccharides (FOS) may have potential benefits, since they exhibit many soluble dietary fibre-like properties. Foods currently available for weaning infants are generally low in fibre content and lack these potential benefits. Data documenting tolerance of FOS in weaning foods are greatly lacking. Our present objective was to evaluate the tolerance and gastrointestinal effects of FOS-supplemented infant cereal used as a daily addition to the diet of healthy infants. Healthy infants were randomly assigned to receive either $0.75 \mathrm{~g}$ FOS per serving of cereal or placebo for $28 \mathrm{~d}$. The primary outcome of interest was gastrointestinal tolerance, which was assessed by daily parental reporting of functional variables for $28 \mathrm{~d}$, including stool patterns and signs and symptoms of gastrointestinal tolerance. Secondary outcomes were also measured including: cereal intake (g cereal and g FOS/d), stool pH, changes in anthropometric measurements and adverse events. The study population included a total of fifty-six infants, age range 16.2-46.2 weeks with a mean age of 32.5 (SD 8.9) weeks; twenty-nine infants were randomized to the control group (age 31.8 (SD 9.0) weeks) and twenty-seven to the FOS-supplemented group (34.7 (SD 8.9) weeks). Average daily total intake per infant and average intake per serving were similar in both groups. Average FOS consumption was 0.74 (SD 0.39) $\mathrm{g} / \mathrm{d}$ and as high as $3.00 \mathrm{~g} / \mathrm{d}$. Stool consistency was less likely to be described as 'hard', and more likely to be described as 'soft' or 'loose', in the FOS $v$. control group. The mean number of stools per infant was 1.99 (SD 0.62) per d in the FOS-supplemented group compared with 1.58 (SD 0.66) in the control group $(P=0.02)$. There were no differences between the groups in reporting for crying, spitting-up or colic. No differences were found for stool $\mathrm{pH}$. FOS-supplements added to cereal were well tolerated in doses of up to $3.00 \mathrm{~g} / \mathrm{d}$. FOS consumption led to more regular and softer stools, without diarrhoea, as well as less-reported frequency of symptoms associated with constipation such as hard stools or skipped days without stool. The present study is one of few studies documenting tolerance to increased fibre intake in the form of FOS as part of a weaning food.
\end{abstract}

Fructo-oligosaccharides: Stool characteristics: Infant cereal: Weaning foods

Fructo-oligosaccharides (FOS) are a mixture of oligosaccharides, consisting of glucose linked to fructose units by $\beta 1,2$ glycosidic linkages; they are indigestible and highly fermentable (Roberfroid \& Delzenne, 1998). They are naturally present in varying concentrations in many foods such as wheat, banana, asparagus and garlic, and can also be produced enzymatically (Spiegel et al. 1994; Van Loo et al. 1995).

Consumption of FOS has potential benefits, since FOS is a soluble dietary fibre and is resistant to digestion by endogenous enzymes, reduces faecal $\mathrm{pH}$, increases the water-holding capacity of stool and faecal weight and decreases gastrointestinal transit time (Hidaka et al. 1986). Like other soluble fibres, FOS has been shown to improve moderate constipation, reduce plasma cholesterol and triacylglycerol and to moderate glucose absorption in adults (Hidaka et al. 1986; Rumessen et al. 1990). In addition, colonic fermentation of FOS in infants can have prebiotic effects by selectively stimulating the growth of probiotic bacteria, such as Bifidobacterium, the predominant flora of breast-fed infants (Boehm et al. 2002; Moro et al. 2002). Bifidobacterium has been shown to be associated with gastrointestinal and systemic health benefits (Modler et al. 1990; Gibson et al. 1994; Garleb et al. 1996).

There are few studies documenting paediatric tolerance of FOS-supplemented foods. Cadranel \& Coussement (1995) reported a preliminary study of children, 10-13 years of age, who received daily supplementation of FOS, and documented no abnormal stools, diarrhoea or gastrointestinal side effects. The authors concluded that $\leq 9 \mathrm{~g} \mathrm{FOS} / \mathrm{d}$ are well tolerated by children in this age group. In a double-blind crossover trial of FOS, Van den Heuvel et al. (1999) also reported no difference in gastrointestinal symptoms in adolescents consuming $15 \mathrm{~g} \mathrm{FOS} / \mathrm{d}$.

Overall, there are limited results on infant intake and tolerance of FOS when derived from supplemental sources. Specifically, no published results are available on intake 
or tolerance of FOS in infants consuming weaning foods, such as a complete baby cereal (a rice cereal with ingredients of formula) containing FOS. The purpose of the present controlled trial was to evaluate the tolerance, intake and changes in stool characteristics in infants given FOS-supplemented cereal as an addition to a regular weaning diet.

\section{Methods}

\section{Subjects and study design}

A sample of healthy term infants aged 4-12 months was recruited from the Baltimore (MD, USA) metropolitan area. Participating families received a gift certificate, equivalent to a 1-month supply of nappies at a children's retail store, in exchange for participation. The institutional review board of the Johns Hopkins Medical Center (Baltimore, MD, USA) approved the study protocol.

All infants were former term newborns ( $\geq 37$ weeks gestation and $\leq 42$ weeks gestation) with birth weights $\geq 2500$ and $\leq 4200 \mathrm{~g}$. Infants were aged 4-11 months at enrolment and had demonstrated tolerance of rice cereal and standard milk-based, lactose-containing infant formula for a minimum of 1 week based on the recall of the parent or caregiver. Infants from multiple gestations were eligible; however, siblings could not be enrolled in the trial at the same time.

The following groups of infants were excluded from the study population: infants with major congenital birth deformities, acute illness at enrolment, chronic conditions affecting food intake or metabolism, infants readmitted to a hospital after birth for more than $2 \mathrm{~d}$, infants receiving more than one feeding of breast milk per $\mathrm{d}$ and infants participating in another clinical study.

Subjects were randomly assigned by block allocation to receipt of either the FOS-supplemented or control cereal. Cereal was prepared by the parent or caregiver using printed label instructions. Addition of other foods to the cereal was permitted as long as steps were taken to document and measure the actual cereal intake. Infants were offered a minimum of one serving of the assigned cereal per $\mathrm{d}$ for $28 \mathrm{~d}$.

\section{Trial diets}

The cereals, supplied by Nestlé USA (Glendale, CA, USA), are intended for feeding infants aged 4-12 months. One serving size ( $25 \mathrm{~g}$ cereal) is approximately one-third of a cup (US measure). Each serving provides $460 \mathrm{~kJ}$ $(110 \mathrm{kcal})$ with $3 \mathrm{~g}$ protein and $2.5 \mathrm{~g}$ fat. Protein sources are from milk and rice, and fat sources are provided by palm olein, soyabean oil, coconut oil and milk fat. The total carbohydrate content was $16 \mathrm{~g}$ per serving, comprising hydrolysed rice, rice flour, lactose and either soluble fibre as FOS or the equivalent amount of maltodextrin. The intervention group received infant cereal (Nestlé Carnation Premium Baby Cereal ${ }^{\circledR}$; Nestlé USA) containing 0.03 g FOS/g cereal $(0.75 \mathrm{~g}$ FOS $/ 25 \mathrm{~g}$ serving of cereal). The control group received the same cereal but with $0.03 \mathrm{~g}$ maltodextrin in place of FOS $/ g$. Both cereals were in powder form and packaged identically except for differences in canister label colour and product code number.

\section{Data collection}

The primary outcome of interest was cereal tolerance, which was assessed daily by parent or caregiver evaluation and reporting of gastrointestinal symptoms for a total $28 \mathrm{~d}$ of participation. The following measures were used to assess tolerance: stool frequency, stool colour and consistency, infant flatus, occurrence of vomiting, spitting-up, crying and colic and/or abdominal cramps. The following secondary outcomes were also included: cereal intake ( $\mathrm{g}$ cereal/d and $\mathrm{g}$ FOS/d), stool fermentation based on stool $\mathrm{pH}$, changes in anthropometric measurements and adverse events.

\section{Tolerance measures}

A standardized daily record log was supplied to each parent or caregiver and reviewed by the study coordinator during phone interviews each week. For each stooling event, the parent or caregiver characterized the predominant stool colour as 'brown', 'green', 'yellow' or 'black' and stool consistency as either 'hard (like clay or harder)', 'firm (formed like Play Doh ${ }^{\circledR}$ )', 'soft (like pudding)', 'loose (like applesauce)' or 'watery (like urine)'. The stool consistency descriptions provided to parents were utilized in previous trials by the authors and appeared to be both understandable by the parent or caregiver and internally consistent (Abi-Hanna et al. 1998) although no formal validity tests were utilized.

For assessment of infant flatus, vomiting, spitting-up, crying and colic and/or abdominal cramps, for each day the parent or caregiver recorded if the events occurred 'less than usual', 'about usual' or 'more than usual'. Spitting-up was defined as: 'the effortless return of small amount of swallowed food, usually a mouthful'. Vomiting was defined as 'the forceful return of larger amounts of food with more complete emptying of the stomach'.

\section{Secondary outcomes}

For each offering of cereal, the parent or caregiver used a standardized procedure to record the number of scoops of dry cereal, the initial and final volume and what was mixed with the cereal. Based on the cereal intake reported, the amount of FOS consumed at each feeding was calculated.

Following standardized instructions, the parent or caregiver was trained to perform stool $\mathrm{pH}$ tests in the home at weekly intervals up to four times during the study period (days 7, 14, 21 and 28). Parents assessed stool pH by dipping a portion of nitrazine $\mathrm{pH}$ paper into a fresh stool sample within $30 \mathrm{~min}$ of defecation.

\section{Growth data}

Anthropometric measurements were taken by a research associate, using a standardized procedure at baseline (between day -3 and day 0 ) and at study completion 
(day 28). Weights were obtained while the infant was naked, at least $2 \mathrm{~h}$ after feeding or immediately before feeding. The same electronic scale was used at the same time in relation to feeding for enrolment and completion weights. Infant weight was measured twice and the average of the two weights $( \pm 0.01 \mathrm{~kg})$ was documented. Length was measured on a standardized length-board placed on a hard surface. The infants were placed supine in full body extension with feet flexed without shoes. Height was also documented $( \pm 0.001 \mathrm{~m})$. Head circumference was measured approximately $25 \mathrm{~mm}$ above the child's eyebrow, directly over the largest cross-sectional area of the skull. Head circumference was documented to the nearest $1 \mathrm{~mm}$.

\section{Demographic data and clinical course}

Demographic data on maternal age, infant gender, date of birth and anthropometric measures at birth were based on the parent or caregiver's recall. Information on the clinical events during the course of the study, including outpatient or in-patient health care visits and adverse events, was also collected. An adverse event was defined as any untoward occurrence in the infant such as illnesses or unusual signs or symptoms perceived or reported by the parent or caregiver during the course of the study.

\section{Analysis}

Sample size ( $n 25$ per group) was calculated on the basis of our own preliminary data to detect a $25 \%$ difference between the study groups in the alternative hypothesis for any of the primary outcomes on tolerance (i.e. stool frequency, colour and consistency) at $5 \%$ significance level and $80 \%$ power.

Data were analysed based on the intention-to-treat principle and measured tolerance and intake if: (1) baseline and at least $3 \mathrm{~d}$ of intake measurements were available; (2) the data supplied by the parent were internally consistent and plausible. Infants in the population to have efficacy evaluated were included if they met all inclusion criteria, received the study diet for more than $3 \mathrm{~d}$ and had stool measurements taken.

Summary statistics such as gender, age, birth weight and baseline anthropometric measures of the study subjects for both groups were used to assess the baseline characteristics.

Mantel-Haenszel $\chi^{2}$ tests and contingency tables were used to evaluate the differences in the categorical primary outcomes between the two randomly assigned groups. The event frequencies of these variables were computed by counting the number of subject days per group for each event category. Student's $t$ tests were used to evaluate the differences in the mean values of the continuous anthropometric variables between these groups. To assess the differences in colic, crying, spitting-up, vomiting and flatus, we computed the proportion of days for an infant in each group having 'more than usual' for each of the symptoms described earlier, and compared the mean values of these proportions between the two groups using Student's $t$ tests.

\section{Results}

A total of fifty-seven infants were recruited. One subject was excluded from the analysis due to internally inconsistent data supplied by the parent. Of the remaining fifty-six infants, twenty-seven were randomly assigned to the experimental cereal and twenty-nine to receive the control cereal. For each day of the study, we collected daily information for each subject for a total of 1471 observation days.

Four control subjects did not complete the full $28 \mathrm{~d}$ of the study for the following reasons: voluntary withdrawal onto finger foods and infant refusal to consume the cereal $(n 2)$; caregiver non-compliance with documentation protocol ( $n$ 1); increased perceived 'fussiness' by parent which led to withdrawal ( $n$ 1). From the experimental group, one subject dropped out due to parental non-compliance with the study protocol (i.e. mother did not offer weaning cereal for $>3 \mathrm{~d}$ ). The five infants with premature withdrawal from the study completed a median of $7 \mathrm{~d}$ of the study.

There were no significant differences in demographic or anthropometric data between the two groups (Table 1).

\section{Tolerance}

The experimental cereal was well tolerated based on our analysis of 2723 stooling episodes recorded in 1464 observation days. Infants receiving FOS had more frequent stools of regular consistency.

\section{Stool frequency}

Infants in the FOS-supplemented group had a greater frequency of having more stools per $d$ than the control group. The mean number of stools per infant was 1.99 (SD 0.62) per $\mathrm{d}$ in the FOS-supplemented group compared with 1.58 (SD 0.66) in the control group $(P=0.02)$. The number of parent-reported stools ranged from zero to six per $\mathrm{d}$ for the control group and zero to five per $\mathrm{d}$ for the group receiving FOS. The overall distribution of the number of stools per $\mathrm{d}$ for both groups is depicted on Fig. 1 and suggests a shift towards an increased number of stools per $\mathrm{d}$ for the FOS-supplemented group. However, for both groups, there were less than four stools per $\mathrm{d}$ for almost all $(99 \%)$ observation days. Infants receiving FOS had one-third of the number of observed days with no stool (15 v. $46 \mathrm{~d})$. There was no difference in the quantity of juice intake between the two groups to account for potential differences in stool frequency $(P=0 \cdot 29)$.

\section{Stool consistency}

The parent-reported stool consistency for each sequential stooling event of the day was compared. For infants receiving FOS-supplemented cereal, stool consistency was less likely to be described as 'hard' and more likely to be described as 'soft' or 'loose', but not watery. This finding was noted for only the first $\left(\chi^{2} 11 \cdot 14, P<0 \cdot 01\right)$ and second stooling event $\left(\chi^{2} 14.70, P<0.01\right)$ of the day. There were only twenty-one stooling episodes that were 
Table 1. Baseline characteristics of the subjects according to the study group (Mean values and standard deviations)

\begin{tabular}{|c|c|c|c|c|}
\hline & \multicolumn{2}{|c|}{$\begin{array}{l}\text { Unsupplemented control } \\
\text { group }(n 29)\end{array}$} & \multicolumn{2}{|c|}{$\begin{array}{l}\text { FOS-supplemented } \\
\text { group ( }(n 27)\end{array}$} \\
\hline & Mean & SD & Mean & SD \\
\hline \multicolumn{5}{|l|}{ General characteristics } \\
\hline Male & $17 \dagger$ & $59 \ddagger$ & $13 \dagger$ & $48 \ddagger$ \\
\hline Age (weeks) & 31.8 & 9.0 & $34 \cdot 7^{\star}$ & 8.9 \\
\hline Birth weight $(\mathrm{kg})$ & 3.58 & 0.51 & $3.46^{*}$ & 0.30 \\
\hline Birth length $(\mathrm{m})$ & 0.513 & 0.026 & $0.517^{\star}$ & 0.023 \\
\hline Gestational (weeks) & 39.6 & 1.4 & $39 \cdot 6^{*}$ & 1.3 \\
\hline Age when weaning initiated (weeks) & $16 \cdot 0$ & 3.5 & $16 \cdot 3^{*}$ & 4.8 \\
\hline Length of time on breast milk (weeks) & 9.0 & $9 \cdot 3$ & $12 \cdot 1^{*}$ & 13.5 \\
\hline
\end{tabular}

FOS, fructo-oligosaccharide.

Mean values were significantly different from those of the control group: ${ }^{*} P<0.05$. $\dagger n$.

$\ddagger \%$.

described as 'watery'. Twenty of these twenty-one episodes $(95 \%)$ were reported for infants receiving the control cereal.

\section{Other gastrointestinal variables}

Although the frequency and consistency of stool was different for both groups, the distribution for the parentreported stool colour was the same for both groups. This finding was consistent for each stool of the day.

Parents described each of the following symptoms: infant flatus, presence of vomiting, spitting-up, crying and colic and/or abdominal cramps as 'less than usual', 'about usual' or 'more than usual' for each day of the study. There were no differences in the mean number of days per infant that these symptoms were 'more than usual' for crying, spitting-up or colic.

\section{Secondary outcomes}

Daily and serving consumption. The average daily total intake of cereal per infant was similar in both groups $(P=0 \cdot 72)$. Infants receiving FOS-supplemented cereal had a similar average daily total intake (35.0 (SD 22.5) g dry cereal/d per infant) of cereal compared with infants taking the control cereal (35.0 (SD 27.5) g dry cereal/d per infant). The amount consumed by the experimental group was equivalent to 1.05 (SD 0.68) g FOS/d per infant. The FOS group had a total single-day intake range of zero to four servings ( $25 \mathrm{~g}$ per serving) of cereal (up to $3.00 \mathrm{~g}$ FOS), compared with zero to nine servings for the control group.

The average consumption of cereal per serving was similar for both groups $(P=0.92)$. For the FOS group, the average consumption per serving was 0.98 (SD 0.52) scoops of cereal with a range of zero to four scoops. This amount is equivalent to 0.74 (SD 0.39) g FOS with a range of 0.00 to $3.00 \mathrm{~g}$ FOS per serving. For the control group, the average consumption per serving was 0.97 (SD 0.46) scoops with a range of zero to three scoops per serving.

Stool $\mathrm{pH}$. The average stool $\mathrm{pH}$ for each infant was compared to assess any affect on fermentation: there was no difference between the groups. Average stool $\mathrm{pH}$ was 6.1 (SD 0.77) for infants receiving FOS and 6.4 (SD 0.94) for infants receiving the control cereal $(P=0.13)$.

Adverse events. There were no serious adverse events reported in either group. However, for non-serious adverse

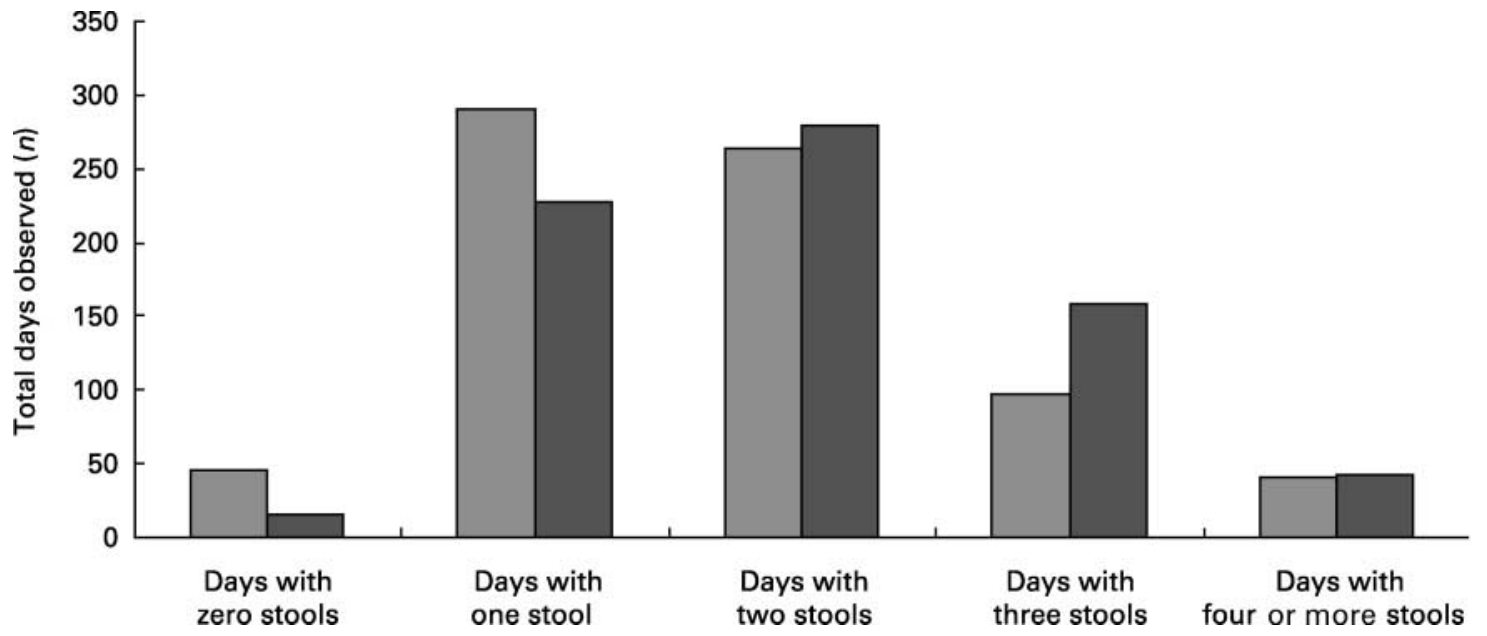

Fig. 1. Stool frequency $(n$ per d) reported by the parent or caregiver of healthy infants receiving $0.75 \mathrm{~g}$ fructo-oligosaccharide per serving of cereal $(n 27 ;-)$ or a placebo $(n 29 ; \mathbf{\square})$ for 25 d. For details of subjects, cereals and procedures, see Table 1 and p. 582. 
events, seventeen experimental families reported twentyfour non-serious adverse events, compared with sixteen control families reporting twenty-one non-serious adverse events. The non-serious adverse events and their frequencies are listed in Table 2. Only one of the non-serious adverse events (loose stools) was considered 'probable in relation to the test product'; however, this subject was subsequently found to be receiving the control cereal.

During the study period, ten infants saw their primary care physician. Seven of the ten visits were for routine well-baby examinations. The remaining three visits (two infants on FOS-supplemented cereal) were for ambulatory illnesses and did not lead to hospitalization.

Effect on growth. Anthropometric measurements taken at baseline (between days -3 and 0) were compared with those obtained at study completion (day 28). Changes in weight during this time period were +0.56 (SD 0.23$) \mathrm{kg}$ for the FOS-supplemented group and +0.54 (SD 0.24) $\mathrm{kg}$. Infant recumbent length also increased from baseline by 20 (SD 13) and 16 (SD 12) mm for FOS-supplemented and control groups respectively. There was no significant difference in growth between the two groups.

\section{Discussion}

The present study is the first to be published documenting tolerance to increased fibre in the form of FOS in infants taking weaning foods, such as cereal. In addition, the present study is one of few studies, in general, documenting tolerance to FOS. Infants taking the FOS-supplemented cereal had more frequent and softer stools, without any reported diarrhoea, in infants taking up to $3.00 \mathrm{~g}$ FOS/d. There were no differences in flatus and colic and/or abdominal cramps according to the parents.

Currently, there is a lack of scientific data available to define appropriate fibre intakes for infants and children $<2$ years of age (American Academy of Pediatrics, 1995; American Dietetic Association, 1997). However, research is currently underway to identify the long- and short-term benefits and risks, quantity and types of fibres that would be appropriate for this age group (Williams, 1995; Weaver, 2000). The current American Academy of Pediatrics' recommendation for fibre intake in healthy children $>2$ years of age is to consume the amount in $g$ equal to or greater than their age (in years) plus $5 \mathrm{~g} / \mathrm{d}$ (Williams, 1995; Williams \& Bollella, 1995; Williams et al. 1995;
Hampl et al. 1998). Therefore, at 3 years of age, fibre intake would be eight $(3+5) \mathrm{g} / \mathrm{d}$ and incrementally increase to $25 \mathrm{~g} / \mathrm{d}$ by age 20 years (Dwyer, 1995; Williams \& Bollella, 1995; Williams et al. 1995). This level of fibre intake is within a range thought to provide known health benefits without compromising either mineral balance or energy intake in children $\geq 3$ years of age (American Academy of Pediatrics, 1995; Dwyer, 1995).

Fibre intake recommendations based on nutritional surveys in children $<2$ years old have been proposed. Agostoni et al. (1995) recommend that the weaning diet of infants aged 6-12 months should contain at least $5 \mathrm{~g}$ soluble and insoluble fibre/d and $7 \mathrm{~g}$ fibre/d by the age of 2 years. Alexy et al. (1999) assessed the macronutrient intake of $>350$ weaning infants and documented that weaning foods routinely included fruits, vegetables and grains that contain dietary fibre and FOS. His study suggested that fibre intake was highest at the age of 1 year, ranging from 8 to $10 \mathrm{~g} / \mathrm{d}$ (Alexy et al. 1999).

Foods with added dietary fructan such as FOS are widely available in many industrialized countries and infant formula fortified with FOS has been safely consumed by thousands of newborn infants and toddlers in Japan since 1989 (Yamamoto \& Yonekubo, 1993). Although there are many clinical trials with human subjects documenting the safety and tolerance to FOS in adults (Hidaka et al. 1986; Garleb et al. 1996), paediatric studies are limited to infants taking formula only (Yamamoto \& Yonekubo, 1993; Boehm et al. 2002; Moro et al. 2002) and older children or adolescents (Cadranel \& Coussement, 1995; Van den Heuvel et al. 1999).

Yamamota \& Yonekubo (1993) surveyed 20742 infants $<5$ months of age in Japan and found that infants exclusively fed FOS-supplemented formula represented onefifth of the study population (Yamamoto \& Yonekubo, 1993). They noted no differences between infants exclusively fed FOS-supplemented formula $v$. other babies for growth or the mother's perception of their infant's health. FOS intakes were 3.0 and $4.2 \mathrm{~g} / \mathrm{d}$ for the 50th and 90th percentile respectively (Yamamoto \& Yonekubo, 1993). In our present study, with a slightly older subject population (mean age 8.3 months) on a weaning diet, FOS intakes were 0.75 and $2.25 \mathrm{~g} / \mathrm{d}$ for the 50 th and 90th percentile respectively.

The current study found that infants tolerated up to $3.00 \mathrm{~g}$ FOS/d, well below the suggested or estimated fibre

Table 2. Non-serious adverse events reported $(n \text { per } 28 \mathrm{~d})^{\star}$

\begin{tabular}{lrr}
\hline Description & $\begin{array}{c}\text { Unsupplemented } \\
\text { control group }(n \text { 29) }\end{array}$ & $\begin{array}{r}\text { FOS-supplemented } \\
\text { group }(n \text { 27) }\end{array}$ \\
\hline Cold symptoms (cough, nasal congestion) & 12 & 12 \\
'Fussy' behaviour associated with teething & 1 & 3 \\
General gastrointestinal complaints (vomiting, loose stools) & 4 & 24 \\
Fever with viral cold symptoms & 2 & 3 \\
Fever without other symptoms & 2 & 1 \\
Otitis media & 0 & 1 \\
Coxsackie virus infection & 0 & 1 \\
'Eye infection' & 0 & 1 \\
\end{tabular}

FOS, fructo-oligosaccharide.

* For details of subjects, supplements and procedures, see Table 1 and p. 582. 
intake for healthy weaning infants. In the study population, FOS intake was measured from the cereal supplement only. It is possible that FOS was also consumed in other dietary sources.

Reported side effects of soluble fibre supplementation include an increase in intestinal gas and discomfort. This phenomenon tends to occur when there is a significant and acute increase in fibre intake, leading to increased fermentation, probably as intestinal microflora adapt to new substrates. However, our present results showed no difference in these variables for the two groups. In addition, in the present study FOS was included as part of a mixed diet, not as an isolated supplement and this may mitigate possible gastrointestinal intolerance.

In young children, high-fibre diets can potentially reduce energy density and energy intake, thus resulting in poor growth. However, this bulking effect is associated primarily with insoluble fibres (Dwyer, 1995; Williams \& Bollella, 1995). The inclusion of modest amounts of soluble fibre, such as those found in fruits, vegetables, legumes and certain grains, would be less likely to result in growth effects (Dwyer, 1995). In addition, the results of our present study revealed no differences in growth for the two groups.

There are some limitations to the present study. The parental assessment of stool consistency was standardized and uniformly administered by trained personnel: this allows comparison between the groups. However, the tool per se has not been independently validated. The sample size was relatively small and may not allow detection of significant differences in secondary outcomes such as growth or other general health outcomes. However, the study duration was sufficient to identify signs of intolerance or other gastrointestinal effects to FOS-supplemented cereal. Moro et al. (2002) also reported that FOS-supplemented formula in term infants had no influence on the incidence of side effects (crying, regurgitation, vomiting) or growth during a $28 \mathrm{~d}$ study period. Longer studies would be helpful to document longer-term effects on growth. Such studies may also detect changes in stool $\mathrm{pH}$, which may occur secondary to FOS effects on gut flora and therefore may require a longer time to become evident.

\section{Implications}

More scientific data are needed to define optimal fibre intakes for infants and children $<2$ years of age, longand short-term benefits, as well as quantity and types of fibres that would be most appropriate for this age group (in particular, for fibres that may confer potential health benefits such as the prebiotic effect of FOS).

The present study carefully documents adequate tolerance to a range of intake of FOS-supplemented cereal product. This supplementation provides a practical, age-appropriate and developmentally appropriate approach to confer some of the benefits of soluble fibre in infants and toddlers.

The results of the present study suggest that FOSsupplemented cereal is tolerated in infants with positive effects on bowel habits (improved stool regularity and consistency). Further studies are needed to examine if these findings are applicable to other populations and if FOS supplementation in weaning foods leads to other measurable long- and short-term benefits for infants.

\section{Acknowledgements}

This study was funded in part by a grant from Nestlé Nutrition Institute (Glendale, CA, USA). The authors would like to acknowledge $\mathrm{Dr}$ Colin $\mathrm{Wu}$ for his input in the analysis of data.

\section{References}

Abi-Hanna A, Moore N, Yolken R \& Saavedra J (1998) Long term consumption of infant formulas with live probiotic bacteria; safety and tolerance. J Pediatr Gastroenterol Nutr 7, A84.

Agostoni C, Riva E \& Giovannini M (1995) Dietary fiber in weaning foods of young children. Pediatrics 96, 1002-1005.

Alexy U (1999) Macronutrient intake of 3-to-36 month old German infants and children: results of the DONALD study. Dortmund Nutritional and Anthropometric Longitudinally Designed Study. Ann Nutr Metab 43, 14-22.

American Academy of Pediatrics (1995) A summary of conference recommendations on dietary fiber in childhood. The role of dietary fiber in childhood. Pediatrics 96, 1023-1028.

American Dietetic Association (1997) Position of ADA: Health implications of dietary fiber. J Am Diet Assoc 97, 1157-1159.

Boehm G, Casetta P, Jelinek J, Negretti F, Stahl B \& Marini A (2002) Supplementation of bovine milk formula with an oligosaccharide mixture increases counts of faecal bifidobacteria in preterm infants. Arch Dis Child 86, F178-F181.

Cadranel S \& Coussement P (1995) Tolerance study with oligofructose for school children. Proceedings of First Orafti Research Conference. Hospital Universitaire des Enfants Reine Fabiola. Brussels, Belgium.

Dwyer JT (1995) Dietary fiber for children: How much? Pediatrics 96, 1019-1022.

Garleb KA, Snook JT, Marcon MJ, Wolf BW \& Johnson WA (1996) Effect of fructooligosaccharides containing enteral formulas on subjective tolerance factors, serum chemistry panels, and faecal bifidobacteria in healthy male adult subjects. Microb Ecol Health Dis 9, 279-285.

Gibson GR, Willis C \& Van Loo J (1994) Non-digestible oligosaccharides and bifidobacteria: implications for health. Int Sugar J 96, 381-387.

Hampl JS, Betts NM \& Benes BA (1998) The 'age +5 ' rule: comparison of dietary fiber among 4-10 year old children. $J$ Am Diet Assoc 98, 1418-1423.

Hidaka H, Eida T, Takizawa T, Tokunaga T \& Yashiro Y (1986) Effects of fructooligosaccharides on intestinal flora and human health. Bifid Microflora 5, 37-50.

Modler HW, McKellar RC \& Yaguchi M (1990) Bifidobacteria and bifidogenic factors. Can Inst Food Sci Technol J 23, 29-41.

Moro G, Minoli I, Mosca M, et al. (2002) Dose-related bifidogenic effects of galacto- and fructooligosaccharides in formula-fed term infants. J Pediatr Gastroenterol Nutr 34, 291-295.

Roberfroid MB \& Delzenne NM (1998) Dietary fructans. Annu Rev Nutr 18, 117-143.

Rumessen JJ, Bode S, Hamberg O \& Gudmand-Hoyer E (1990) Fructans of Jerusalem artichokes: Intestinal transport, absorption, fermentation, and influence on blood glucose, insulin, and c-peptide response in healthy subjects. Am J Clin Nutr 52, 675-681. 
Spiegel JE, Rose R, Karabell P, Frankos VH \& Schmitt DF (1994) Safety and benefits of fructooligosaccharides as food ingredients. Food Technol 1, 85-89.

Van den Heuvel EGHM, Muys T, van Dokkum W \& Schaafsma G (1999) Oligofructose stimulates calcium absorption in adolescents. Am J Clin Nutr 69, 544-548.

Van Loo J, Coussement P, De Leenheer L, Hoebregs H \& Smits G (1995) On the presence of inulin and oligofructose as natural ingredients in the Western diet. CRC Crit Rev Food Sci Nutr 35, 525-552.

Weaver L (2000) Complex carbohydrates and sugars. Pediatrics 106, 5 .
Williams C (1995) Importance of dietary fiber in childhood. J Am Diet Assoc 95, 1140-1146.

Williams CL \& Bollella M (1995) The role of dietary fiber in childhood. Is a high fiber diet safe for children? Pediatrics 96, 1014-1019.

Williams CL, Bollella M \& Wynder EL (1995) The role of dietary fiber in childhood. A new recommendation for dietary fiber in childhood. Pediatrics 96, 985-988.

Yamamoto Y \& Yonekubo A (1993) A survey of physical growth, nutritional intake, fecal properties and morbidity of infants as related to feeding methods. Shoni Hoken Kenkya 52, 465-471. 